\title{
REFLECTIONS ON \\ THE REVOLUTION AT STANFORD
}

\author{
F.A. Muller
}

\begin{abstract}
We inquire into the question whether the classical ideal of science has been realised by the Model Revolution, initiated at Stanford University during the 1950ies and spread all around the world of philosophy of science - salute P. Suppes. The guiding principle of the Model Revolution is: a scientific theory is a set of structures in the domain of discourse of axiomatic set-theory, characterised by a set-theoretical predicate. We expound some critical reflections on the Model Revolution; the conclusions will be that the philosophical problem of what a scientific theory is has not been solved yet pace P. Suppes. While reflecting critically on the Model Revolution, we also explore a proposal of how to complete the Revolution and briefly address the intertwined subject of scientific representation, which has come to occupy center stage in philosophy of science over the past decade.
\end{abstract}

Keywords: theory, model, classical ideal, structure, being, representation. 


\section{Preamble}

You, dear Reader, are pleased to call again, and with some earnestness, for my thoughts on the late proceedings at Stanford University. I shall not give you reason to imagine, that I think my sentiments of such value as to wish myself to be solicited about them. They are of too little consequence to be very anxiously either communicated or witheld. It was from attention to you, and to you only, that I hesitated at the time, when you first desired to receive them. In the first letter I had the honour to write to you, and which at length I send, I wrote neither for nor from any description of men; nor shall I in this. My errors, if any, are my own. My reputation alone is to answer for them.

This opening paragraph is (with minor modifications) the opening paragraph of Reflections on the Revolution in France, and on the Proceedings in Certain Societies in London Relative to that Event, in a Letter intended to have been sent to a Gentleman in Paris (1790) by the right honourable Edmund Burke. In this long letter to Chames-JeanFrançois de Pont, which detonated the great debate on the French Revolution, Burke defended a Traditionalism that involved a return to an earlier humanity and that contained revolutionary counter-revolutionary prescriptions. Reflections is and always has been a source of inspiration and of arguments for conservative politicians all around the world. Burke's style combines, in varying proportions, a perspicacious rationality, a formidable sonority, a Gothic if not pathetic severity, and a furious irony that frequently glides into a savage sarcasm. ${ }^{1}$

In sharp contrast to all of this, the current paper is not a 'Letter intended to have been sent to a Lady at Stanford University' but is based on a presentation at the engaging conference 'The Classical Model of Science' (Amsterdam, Free University, January 2007); further, it does not advocate a return to an earlier period in the philosophy of science, let alone that it contains 'revolutionary counter-revolutionary prescriptions', and, last but not least, its style is cool, calm and collected. What is analogous in it to Burke's Reflections is that it recognises as revolutionary the change in our thinking about the nature of scientific theories that began at Stanford University about half a century ago under the lead of Patrick Suppes, and that it expounds some reflections on this revolution in the philosophy of science of a critical nature. In addition it also provides some suggestions of how to complete this Model Revolution, rather than to return to the ancien régime. Critically progressive rather than critically conservative is the spirit of this paper.

We begin by providing a succinct summary of what the 'Model Revolution' is all about (Section 2). Then we inquire whether it can be interpreted as achieving the classical ideal of science (Section 3). The subsequent Sections will contain some critical reflections that form the body of this paper, addressing subsequently: first, the relation 
between our theories and the beings they are about (a being is here anything that can exist, and an actual being is anything that does exist; sometimes we drop 'actual' for the sake of brevity; Section 4); secondly, the relation between our theories and the results of experiments and observations (Section 5); and, thirdly, the relation between the models and the concepts and propositions of science (Section 6). We end with an idea of how to complete the Model Revolution (Section 7) and briefly address a topic which is currently in the limelight of philosophy science: scientific representation (Section 8).

\section{The Model Revolution at Stanford}

About half a century ago at Stanford University, in the United States of America, Patrick Suppes and collaborators published, in two papers in Journal for Rational Mechanics and Analysis, a revolutionary axiomatisation of a scientific theory, namely classical particle mechanics Newtonian-style. ${ }^{2}$ Until Suppes' arrived on the stage of philosophy, an axiomatisation of a theory was generally understood in the same sense as this gradually became to be understood in Logic since the appearance of Frege's Begriffschrift in 1979. During the Interbellum, that understanding had been forcefully promulgated by the Logical-Positivistic philosophers of the Vienna Circle; it was inspired by exciting new developments in Logic at the time, which had started in the course of the 19th Century (Frege, Schröder, Russell, Hilbert and others). ${ }^{3}$

In order to characterise some scientific theory $\mathbf{T}$ rigorously, as it is presented in some prominent monograph or review article widely used and referred to by scientists, one had to proceed as follows. ${ }^{4}$

(A) Erect an elementary formal language $\mathcal{L}_{\mathbf{T}}$ (finite lexicon, $\operatorname{LEX}\left(\mathcal{L}_{\mathbf{T}}\right)$, quantification only over all, possibly different sorts of objects, no quantification over predicates; schemata with sentence-variables might be permitted), its lexicon including all predicates that express the 'fundamental concepts' of $\mathbf{T}$. The set $\operatorname{SENT}\left(\mathcal{L}_{\mathbf{T}}\right)$ of the sentences of $\mathcal{L}_{\mathrm{T}}$ is defined inductively in the usual manner. ${ }^{5}$

(B) Erect a formal-deductive apparatus in order to be able to reason rigorously in $\mathcal{L}_{\mathrm{T}}$, i.e. to prove theorems (this fixes the deduction-relation between sentences, abbreviated as usual by: $\vdash$ ).

(C) Collect a number of sentences from $\operatorname{SENT}\left(\mathcal{L}_{\mathrm{T}}\right)$ in set $\operatorname{Ax}(\mathbf{T})$; they are the axioms of $\mathrm{T}$ and should be formalisations of the postulates, principles and laws that characterise $\mathbf{T}$ and are considered not to be deducible from other postulates, principles 
and laws in $\mathbf{T}$. Then pentuple

$$
\left\langle\operatorname{LEX}\left(\mathcal{L}_{\mathbf{T}}\right), \operatorname{SENT}\left(\mathcal{L}_{\mathbf{T}}\right), \operatorname{Ax}(\mathbf{T}), \mathbf{T}, \vdash\right\rangle
$$

is a formal-deductive system, by definition the formalisation of $\mathbf{T}$, where $\mathrm{T}$ is the deductive closure of the axioms: ${ }^{6}$

$\mathrm{T} \equiv\left\{\varphi \in \operatorname{SENT}\left(\mathcal{L}_{\mathbf{T}}\right) \mid \operatorname{AX}(\mathbf{T}) \vdash \varphi\right\}$

Of course one requires that $\mathrm{T}$ be consistent.

Steps (A)-(C) can be taken for mathematical and scientific theories alike ${ }^{7}$; the next steps are for scientific theories only and thus distinguish these from mathematical ones.

(D) Distinguish in the lexicon of $\mathcal{L}_{\mathrm{T}}(\mathrm{A})$ theoretical and observational predicates in order to connect (1) to the results of experiments and observations, e.g. measurements, and collect these predicates in sets $\operatorname{TH}\left(\mathcal{L}_{\mathbf{T}}\right)$ and $\operatorname{OBS}\left(\mathcal{L}_{\mathbf{T}}\right)$, respectively, which thus are both proper subsets of $\operatorname{LEX}\left(\mathcal{L}_{T}\right)$. The axioms of $T(2)$ containing only theoretical predicates are called theoretical postulates and those containing both theoretical and observational predicates - and thus connecting them - are correspondence postulates. Sentences of $\mathcal{L}_{\mathrm{T}}$ consisting of entirely observational predicates, so-called observation sentences, are in principle (but not always in practice) open to verification or falsification, or both; that is to say, whether an observation sentence is true or false can, in principle, be determined just by performing the relevant observation. To make this possible, the observational sub-language of $\mathcal{L}_{\mathrm{T}}$, which contains by definition only all observation sentences without quantifiers, is interpreted (in the standard logical sense) in a specified domain $D_{\mathbf{T}}$ of observable objects (events, things) deemed relevant for $\mathbf{T}$; this also provides, via the correspondence postulates, a so-called partial interpretation of the theoretical sentences.

(E) Let $\mathcal{O}_{t}(\mathbf{T}) \subset \operatorname{SENT}\left(\mathcal{L}_{\mathbf{T}}\right)$ be the set of observation sentences verified by scientists until historical time $t$ that are relevant for $\mathbf{T}$; they are about the members of domain $D_{\mathrm{T}}$ (see D). Call formal-deductive system (1) observationally adequate at $t$ iff $\mathrm{T}$ (2) is (consistent and) every established empirical truth relevant for $\mathbf{T}$ - i.e. every verified observation sentence in $\mathcal{O}_{t}(\mathbf{T})-$ is a member of $\mathrm{T}^{8}$; in other words, iff

$\mathcal{O}_{t}(\mathbf{T}) \subset \mathrm{T} \subset \operatorname{SENT}\left(\mathcal{L}_{\mathbf{T}}\right)$ 
Thus human observations of observable objects, expressed in $\mathcal{L}_{\mathrm{T}}$ constitute the connexion between (i) formal-deductive system (1), which is constructed by us, human beings (as is $\mathbf{T}$ ), and (ii) the concrete actual beings that $\mathbf{T}$ is supposed to be about, of which at most some (but certainly not all) are constructed by us. This observation-nexus codifies the empirical essence of science: without it, there is no science. When the content of $\mathcal{O}_{t}(\mathbf{T})$ grows more varied (difficult to express formally) whilst formal-deductive system (1) remains observationally adequate (3), it also becomes better confirmed. If a number of repeatedly verified sentences in $\mathcal{O}_{t}(\mathbf{T})$ turn out to be inconsistent with $\mathrm{T}$, then system (1) is no longer observationally adequate $(\mathrm{E})$; then it has been empirically falsified: $\mathcal{O}_{t}(\mathbf{T}) \cap \mathrm{T} \neq \mathcal{O}_{t}(\mathbf{T})$, which indeed contradicts (3).

Let us call this answer to the question what theoretical scientific knowledge consists in (the kind of knowledge acquired by science that is stored in theories ${ }^{9}$ ) the FormalLinguistic View (also misleadingly known as 'the syntactic view'), henceforth L-View for brevity. The rigorous reconstrual of $\mathbf{T}$ on the $\mathcal{L}$-View we summarise in the following octuple:

$$
\left\langle\operatorname{LEx}\left(\mathcal{L}_{\mathbf{T}}\right), \operatorname{SENT}\left(\mathcal{L}_{\mathbf{T}}\right), \operatorname{Ax}(\mathbf{T}), \operatorname{OBS}\left(\mathcal{L}_{\mathbf{T}}\right), \operatorname{TH}\left(\mathcal{L}_{\mathbf{T}}\right), \mathbf{T}, \vdash, \mathcal{O}_{t}(\mathbf{T})\right\rangle,
$$

where

$$
\operatorname{LEX}\left(\mathcal{L}_{\mathbf{T}}\right)=\operatorname{OBS}\left(\mathcal{L}_{\mathbf{T}}\right) \cup \operatorname{TH}\left(\mathcal{L}_{\mathbf{T}}\right) \cup \operatorname{MATH}\left(\mathcal{L}_{\mathbf{T}}\right)
$$

is the lexicon of $\mathcal{L}_{\mathrm{T}}$. If $\mathcal{L}_{\mathrm{T}}$ contains object-names, we throw the names of observable objects in $\operatorname{OBS}\left(\mathcal{L}_{\mathbf{T}}\right)$ and those of unobservable objects in $\operatorname{TH}\left(\mathcal{L}_{\mathbf{T}}\right)$ so that $\operatorname{LEX}\left(\mathcal{L}_{\mathbf{T}}\right)$ indeed exhausts the union of the three sets in $(5)$. The third set, $\operatorname{MATH}\left(\mathcal{L}_{\mathbf{T}}\right)$, contains the purely mathematical vocabulary; how much mathematics a scientific employs differs from theory to theory and therefore also $\operatorname{MATH}\left(\mathcal{L}_{\mathbf{T}}\right)$ and $\operatorname{Ax}(\mathbf{T})$ will differ.

The required consistency $(\mathrm{C})$ of $\mathrm{T}$ implies that the part of $\mathrm{T}$ containing only observation sentences is also consistent and thus has a model. In the final and most sophisticated version of the $\mathcal{L}$-View developed by the Logical Positivists it was required that this observational part of T must have a finite model. ${ }^{10}$

The $\mathcal{L}$-View went down due to an accumulation of internal problems and of external criticism - lege infra. In his seminal review article 'The Search for Philosophic Understanding of Scientific Theories', F. Suppe [1974] has described the magnificent rise and fall of this ancien régime. According to some, this programme has ended in failure, and actually was doomed $a b$ ovo - although this last addition requires a crushing amount of wisdom with hindsight; others find priceless pearls of philosophical insight in the remains.

Patrick Suppes was, like the Logical Positivists, inspired by exciting then-new developments in Logic, e.g. the rise of Tarskian model theory in the 1950ies and 1960ies. 
When he axiomatised classical particle mechanics Newtonian-style, he did not follow steps (A)-(E) above of the L-View: he did not create a formal language, he did not describe his deductive apparatus explicitly, he did not erect a formal-deductive system (1), and he did not subdivide predicates into theoretical and observational ones. He did not obtain anything like octuple (4). What Suppes did do when construing scientific theory $\mathbf{T}$ rigorously was something we reconstruct in a manner that will invite comparison with Steps (A)-(E) of the L-View.

(a) Adopt the informal language of axiomatic set-theory (e.g. Zermelo's theory of 1908 with the axiom of choice, ZC) - its formalised version is $\mathcal{L}_{\epsilon}$; it consists of only set-variables and its lexicon contains no names and a single primitive dyadic predicate, the membership-relation $(\in)$; the variables can be expanded with 'primordial elements' aka Urelementen (German).

(b) Adopt elementary predicate logic as the background deductive apparatus in order to be able to reason rigorously in $\mathcal{L}_{\in}$ (prove theorems).

(c) (c.i) Typify the fundamental concepts in $\mathbf{T}$ set-theoretically (whether they are properties, relations, functions, operations, etc. and what their domains and codomains are) and (c.ii) express the postulates, principles and laws that characterise $\mathbf{T}$ set-theoretically; (c.iii) obtain a set-theoretical predicate - frequently called a Suppes-predicate; call its set-extension $T$, which then consists of exactly the sets that are structures meeting the Suppes-predicate - also referred to as models. Whence Suppes' slogan: To axiomatise a theory is to define a set-theoretical predicate. $^{11}$

(d) -

(e) Let $\mathcal{D}_{t}(\mathbf{T})$ be the set of data structures that are obtained until historical time $t$ from the measurement-results of experiments or observations relevant for $\mathbf{T}$, which are extracted from 'the phenomena' that $\mathbf{T}$ is supposed to save. Call $\mathbf{T}$ observationally adequate at $t$ iff for every data structure $\mathfrak{D} \in \mathcal{D}_{t}(\mathbf{T})$, there is some structure (model) $\mathfrak{S} \in \mathbb{T}$ such that $\mathfrak{D}$ is embeddable in $\mathfrak{S}$, where 'embeddability' is broadly construed as some morphism from $\mathfrak{D}$ into (some part of) $\mathfrak{S} .^{12}$

For the sake of rigour, we emphasise that first one has to prove the existence of a convenient set, such as $\mathbf{V}_{\omega+g}$ (a tiny tip of the cumulative hierarchy $\mathbf{V}$ until ordinal level $\omega+g$, where $g$ is the number googol ${ }^{13}$ ), in order to guarantee the existence of set-extension $T$ by the Axiom of Separation, which then licences the Suppes-predicate, say $\tau(\cdot)$, to grab in $\mathbf{V}_{\omega+g}$ :

$$
\mathbf{T} \equiv\left\{\mathfrak{S} \in \mathbf{V}_{\omega+g} \mid \tau(\mathfrak{S})\right\}
$$


so that $\boldsymbol{T} \in \mathbf{V}_{\omega+g}$ and therefore $\boldsymbol{T} \subset \mathbf{V}_{\omega+g}$. When asked to reconstruct some scientific theory, one can simply begin with step (c) and forget steps (a) and (b) because they are always the same. For the axioms of set-theory one takes those of Zermelo's ZC, because on their basis one can construct all mathematics that science needs and ever will need.

The embeddability-relation (e) constitutes the connexion between (i) theory $\mathbf{T}$ and (ii) the phenomena that $\mathbf{T}$ is supposed to describe, to explain or to predict. To save the phenomena is to embed the data structures. The nexus between (i) and (ii) codifies the empirical essence of science: without $i t$, there simply is no science-as-we-know-it. When the content of $D_{\mathbf{T}}(\mathbf{T})$ grows more varied (difficult to express formally) whilst $T$ remains observationally adequate, $T$ becomes better confirmed. If a number of repeatedly obtained data structures in $\mathcal{D}_{t}(\mathbf{T})$ are not embeddable into any structure in $T$, then $T$ is no longer observationally adequate (e); then $T$ has been falsified.

This first sketch of science is, as Suppes warned, not the most accurate picture in which every relevant aspect of science finds a home; more accurate is a hierarchy of structures with the bare data structures at one end and general theory-structures at the other. ${ }^{14}$ We call this view the Informal-Structural View - henceforth S-View for brevity - and the following ordered pair is the reconstrual of $\mathbf{T}$, to be compared with octuple (4) of the $\mathcal{L}$-View:

$$
\left\langle\mathbf{T}, \mathcal{D}_{t}(\mathbf{T})\right\rangle .
$$

Today it seems to have replaced the ancien régime largely. The Model Revolution has succeeded.

Why did the Model Revolution succeed? Why has the $\mathfrak{S}$-View replaced the $\mathcal{L}$ View? For at least six (related) reasons.

I. The first was: internal problems raised by the execution of the $\mathcal{L}$-View and external criticism of its assumptions, notably the assumption of subdividing the vocabulary of every scientific theory clearly in an observational and a theoretical part. ${ }^{15}$

II. Continous with reason I is that the formalisation of scientific theories has been of little help to solve all sorts of philosophical problems about science and raised by science. On the contrary, it evoked all sorts of logical problems that had little do to with the philosophical problems concerning science. Thus B.C. van Fraassen:

The scholastic logistical distinctions that the logical positivists produced - observational and theoretical vocabulary, Craig reductions, Ramsey sentences, firstorder axiomatisable theories, projectible predicates, disposition terms, and all the unholy rest of it - had moved us milles milles de toute habitation scientifique, isolated in our own abstract dreams. ${ }^{16}$ 
III. To execute steps (c) and (e) of the $\mathfrak{S}$-View has turned out to be very much easier than executing steps $(\mathrm{A})-(\mathrm{E})$ of the $\mathcal{L}$-View. The key observation here is due to Suppes: one does not need to formalise in order to be rigorous, for the informal rigour of mathematics, i.e. of set-theory, is just as rigorous as formal rigour is. ${ }^{17}$ Another slogan of Suppes: Mathematics for the philosophy of science, not meta-mathematics. Axiomatic set-theory has turned out to be extremely convenient for characterising scientific theories. Theories of physics, mathematics, chemistry, biology, economy, politics, psychology, linguistics and more have come under its Alexandrian sway. As far as reconstruing scientific theories rigorously is concerned, this programme has met with unprecedented success. ${ }^{18}$

IV. The $\mathfrak{S}$-View puts the model center stage, rather than the theory. In his classic paper 'A Comparison of the Meaning and Use of Models in Mathematics and the Empirical Sciences', which appeared in this very journal about half a century ago, Suppes [1960] argued that his models (i.e. set-structures inhabiting V) could be taken as what working scientists mean by 'model'. Observing further that scientists generally are modelbuilders rather than axiomatisors and theorem-provers, the $\mathfrak{S}$-View seemed to make much more sense of the practice of science than the $\mathcal{L}$-View. During the past decades, N.D. Cartwright and her followers have promulgated a less mathematical and more pragmatic and practical oriented approach to scientific modeling; this approach is a line of development within, or at least given birth to by, the $\mathfrak{S}$-View. ${ }^{19}$

V. The $\mathcal{L}$-View makes a theory heavily dependent on a particular formulation in a specific language $\left(\mathcal{L}_{\mathbf{T}}\right)$, whereas the $\mathfrak{S}$-View provides a characterisation of $\mathbf{T}$ that is not tied up to a particular formulation (see further Section ??).

VI. The part of mathematics that any scientific theory employs needs to be isolated, formalised, axomatised and added to $T$ in the $\mathcal{L}$-View. The $\mathfrak{S}$-View disgards this all and can discard it because, as we asserted earlier, all the mathematics that any scientific theory needs and ever will need can be erected in ZC, which marks our domain of discourse in which we (can) characterise every scientific theory. ${ }^{20}$

\section{Aristotelian Ideals}

In his The Foundations of Mathematics of 1957, E.W. Beth provided a modern characterisation of Aristotle's 'apodeictic science', which has been advocated by the likes of Euclid, Descartes, Arnauld, Newton, Leibniz, Pascal, Kant, Bolzano, Husserl, Frege, Peano, Lesniewski, Russell, Hilbert, Carnap, Tarski, Beth, Montague, ... ${ }^{21}$ W.R. de Jong and A. Betti [2008] have extended this characterisation and baptised it the classical ideal of science'. We shall provide a succinct formulation tailored for present 
purposes. A warning is in order: this classical ideal of science was supposed to be more encompassing than what we nowadays call 'science' (to which the $\mathfrak{S}$-View (7) is geared), and certainly than what we now call 'natural science' (to which the $\mathcal{L}$-View (4) is geared).

We consider an ordered pair

$$
\Pi \equiv\left\langle\Pi_{\mathrm{p}}, \Pi_{\mathrm{c}}\right\rangle
$$

of a set of propositions $\left(\Pi_{p}\right)$ and a set of concepts $\left(\Pi_{c}\right)$. Let us first call $\Pi(8)$ a classical scientific theory iff it meets the following three conditions:

$(\alpha) \Pi$ is about a specific set of actual beings (objects, processes, events, entities, persons, organisms, periods, structures, ...), frequently called its domain or scope.

( $\beta$ ) Some concepts in $\Pi_{c}$ are fundamental; the other concepts in $\Pi_{c}$ are defined in terms of the fundamental ones.

$(\gamma)$ Some propositions in $\Pi_{p}$ are fundamental, often called the principles of $\Pi$; all other propositions in $\Pi_{p}$ follow from these.

Secondly, $\Pi(8)$ is a true and universal and necessary classical scientific theory iff $\Pi$ is a classical scientific theory $(\alpha-\gamma)$ and meets in addition the following two conditions:

( $\delta)$ All propositions in $\Pi_{p}$ are true.

(ع) All propositions in $\Pi_{p}$ are universal and necessary.

In the next two conditions, the existence of actual epistemic agents are needed. Call E our Epistemic community, which consists of all actual human beings having a minimum level of scientific education and are sound of mind). Thirdly, call ordered pair $\langle\Pi, E\rangle$ a classical ideal of science iff $\Pi(8)$ meets conditions $(\alpha-\varepsilon)$ and meets in addition the following two conditions:

(ろ) Every proposition in $\Pi_{\mathrm{p}}$ is known by some members of $E$; every non-fundamental proposition in $\Pi_{\mathrm{p}}$ is known by members of $E$ through its proof.

$(\eta)$ Every concept in $\Pi_{c}$ is known by some members of $E$; every defined concept in $\Pi_{\mathrm{C}}$ is known by members of $E$ through its definition.

Notice that if $\Pi_{p}$ contains infinitely many propositions, conditions $(\zeta-\eta)$ will never be met, unless there are also infinitely many epistemic agents, or unless an omniscient entity is posited and granted membership in $E$ (God, as Bolzano did), or unless 'known' is replaced with 'knowable'. 
Notice too that the classical ideal of science $(\alpha-\eta)$ says nothing about the relation between $\Pi(8)$ and the results of experiments and observations, notably measurements; precisely in this relation we would, certainly since the Scientific Revolution, seek the essence of what we now call science. Hence even if some set $\Pi$ fits the classical profile $(\alpha-\varepsilon)$, we should not conclude that $\Pi$ qualifies as a piece of what we today would call 'scientific knowledge', because something essential has been left out of that classical profile.

De Jong \& Betti [2008] point out that being evident for a proposition, as a guarantee for knowing it ( $\zeta)$, was an indisputable requirement for the fundamental propositions (principles) until the second half of the 19th Century (!) when it comes to geometry, whereafter the decline of this requirement set in. Beth also adds the requirement that the meaning of the concepts in $\Pi_{\mathrm{c}}$ must be clear so as to not require any further explication. ${ }^{22}$ Suppes [1992] has pointed out that Aristotle did not provide a single example of a piece of science that qualifies as achieving the classical ideal by his own lights (ibid., p. 215); the very first piece of science that qualified was Euclid's Elements. We would classify Euclidean geometry today as a piece of pure mathematics. What arguably qualifies as the very first realisation of the classical ideal of science, by our current lights, is Archimedes' theory of static equilibria, although his level of conceptual and axiomatic explicitness does not come close to that of Euclid's (ibid., pp. 217-219).

Since both the $\mathcal{L}$-View (4) and the $\mathfrak{S}$-View (7) are views of scientific theories, rather than of science, and both views are neither about the epistemic status of theories (knowledge) nor about their epistemic relation to us (knowing), they seem epistemically neutral. In spite of this, there is a point in ascertaining whether the $\mathcal{L}$-View and the $\mathfrak{S}$-View meet conditions $(\alpha-\zeta)$, if only to understand why they fail if they fail. Before we do this, we remark that both Views also are neutral, first, about whether scientific theories are true or false, and, secondly, about whether scientific theories are necessary and universal, so that both Views fail to meet conditions $(\delta)$ and $(\varepsilon)$. They fail, however, by remaining silent about these issues, not because they cannot be extended so as to meet these conditions.

Clearly formal-deductive system (1) of the $\mathcal{L}$-View meets conditions $(\alpha-\gamma)$ and is therefore a classical scientific theory when we take the primitive predicates of $\mathcal{L}_{\mathrm{T}}$ to express the fundamental concepts in $\Pi_{\mathrm{c}}$ and syntactic definability as 'definability' (in $\beta$ ), and when we take the sentences of $\mathcal{L}_{\mathrm{T}}$ to express the propositions in $\Pi_{\mathrm{p}}$ and the axioms of $T$ (2) to express the principles in $\Pi_{p}$ (in $\gamma$ ). Whether $T$ (6) of the $\mathfrak{S}$-View meets conditions $(\alpha-\gamma)$ is highly problematic, because $T$ is not a set of propositions and concepts (but a set of set-structures) whilst the predicate 'classical ideal of science' only applies such sets $(\Pi)$. However, if we consider the set-theoretical renderings of the 
fundamental concepts of $\mathbf{T}$ as expressions of the fundamental concepts in $\Pi_{c}$, and if we consider the characterisation conjuncts in the definition of the set-theoretical predicate whose set-extension is $T$ (6) as expressions of the fundamental propositions in $\Pi_{p}$, then there is a case to be made for the claim that $T$ also qualifies as a classical scientific theory.

The condition of the classical ideal of science of which we have so far remained silent is the beings condition $(\alpha)$. We have taken it for granted that in the $\mathcal{L}$-View $(4)$, the specification of the subject-matter of $T$ proceeds semantically: whatever the variables of $\mathcal{L}_{\mathbf{T}}$ range over and to whatever the predicates of $\mathcal{L}_{\mathbf{T}}$ apply are the beings that $\mathbf{T}$ is about (unintended models of $\mathrm{T}$ (2) now appear at the horizon, we quickly change course). For the $\mathfrak{S}$-View we cannot take something similar for granted, because $T$ (6) does not consist of propositions that are about something; the set-theoretical predicate $\tau(\cdot)$ that defines $T$ is an open sentence in $\mathcal{L}_{\in}$ and therefore is about sets only, inhabitants of $\mathbf{V}$, not of the world. But surely scientific theories are about concrete actual beings in the world and not about abstract sets in the domain of discourse $\mathbf{V}$ of set-theory.

To conclude, the only good news for propounders of the classical ideal of science is that formal-deductive system (1) of the $\mathcal{L}$-View (4) qualifies as a classical scientific theory. Bad news is that the classical ideal of science says nothing about the relation between theory and observation, whereas precisely here resides the essence of what we call science. There have been historical attempts to change the characterisation of the classical ideal of science so as to include observations and measurements, but that will then be a characterisation of a different concept, as different as Galilean-Newtonian physics was from its Aristotelian-scholastic progenitors. Perhaps one could argue that the specific failure of $T$ of the $\mathfrak{S}$-View (7) to qualify as a classical scientific theory $(\alpha-\gamma)$ does not spell trouble for the classical ideal of science, but rather spells trouble for the $\mathfrak{S}$-View. This is indeed what we shall argue in the next Section, where we expound our first critical reflection on the Model Revolution at Stanford.

\section{Deep Diving for the Beings}

In the previous Section, we emphasised that the set-theoretical predicate $\tau(\cdot)$ that defines $T$ is an open sentence in the language of pure set-theory $\left(\mathcal{L}_{\in}\right)$; therefore it is literally about sets and not about anything else. Furthermore, theory $T$ (6) is a set of structures in $\mathbf{V}$. How can any scientific theory $\mathbf{T}$ thus reconstructed be about any concrete being in the world, say B (of 'Being')? Ontological variable ' $B$ ' may have as values our solar system, Einstein's brain, the French Revolution, a human cell, the Big Bang, a Uranium nucleus, the entire universe, the market economy of Russia after the fall of the 
Berlin wall, the population of lions and zebras in Kenia, an earthquake in Turkey, and so forth - all concrete actual beings in the wide sense in which we are using this term. The fact that the $\mathfrak{S}$-View does not meet the beings condition $(\alpha)$ of the classical ideal of science counts against this View, not against the classical ideal. Yet Suppes argued, as we have also mentioned previously, that his set-structures could be taken as what working scientists mean by 'model', which is exactly the reason why the structures are aka models (Section 2). But any model in science is a model of some concrete actual being B. What we know about the modelled beings we know via our scientific models and theories; the epistemic aim of science is the production of knowledge of actual beings. About this putative connexion, between the model $\mathfrak{S} \in \boldsymbol{T}$ and actual beings (B), the $\mathfrak{S - V i e w ~ p a s s e s ~ o v e r ~ i n ~ s i l e n c e . ~}$

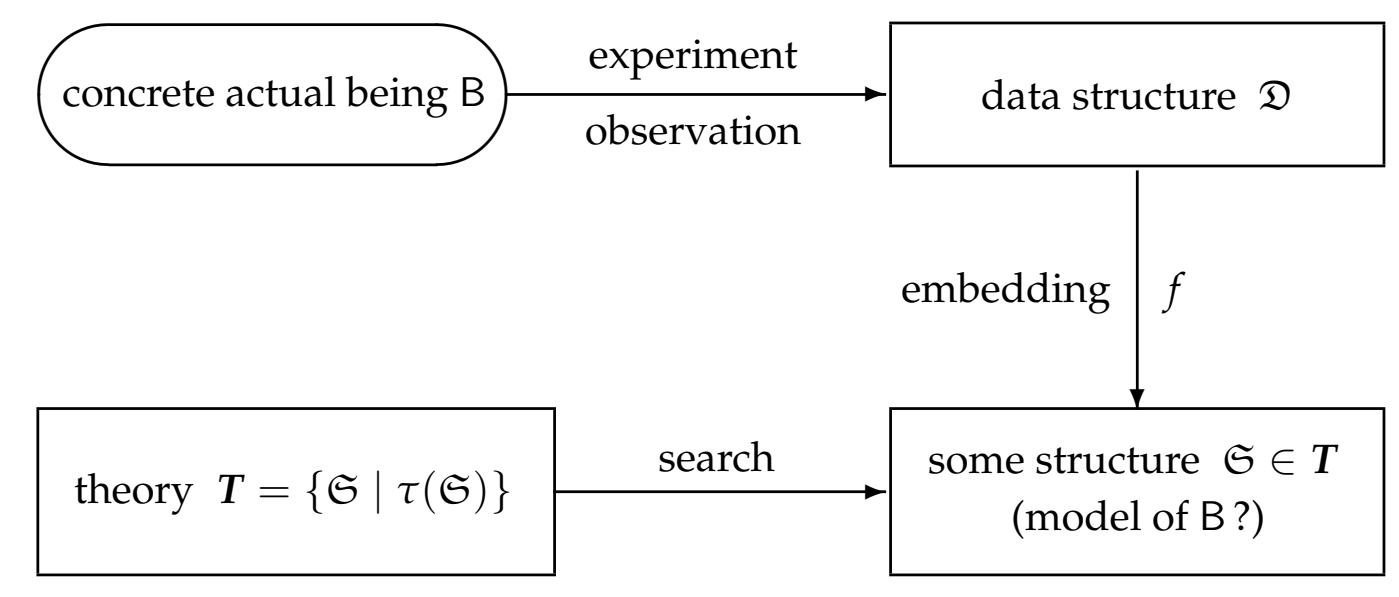

Figure 1: The Informal-Structural View (7), or S-View

Do beings actually enter the picture in the $\mathfrak{S}$-View at all? They do when we draw a picture (see Figure 1). There we can find a place for the beings. On the one hand they are what all experiments and all observations in science are about; measurements are the result of the interaction between concrete actual beings and our instruments of experimentation and observation, which are also concrete actual beings. On the other hand, their role in the $\mathfrak{S}$-View is not one played somewhere on stage for all of us to spot and to marvel at, let alone in the limelight drawing attention, but somewhere backstage, as somehow responsible for the phenomena, from which data structures $\mathfrak{D}$ are extracted, because the phenomena are the result of interaction between concrete actual beings and us, human beings. In contrast, the data-structures are present on stage, showing off in the limelight. As soon as some data structure $\mathfrak{D}$ is obtained, we 
can forget all about the concrete actual beings B at hand, or so it seems. The S-View says next to nothing about how the models $\mathfrak{S}$ (the theory $T$ ) are related to the concrete beings $B$ they are supposed to provide knowledge of. As we concluded above, the $\mathfrak{S}$-View passes over in silence.

The best one could say is that a data structure $\mathfrak{D}$ seems to act as simulacrum of the concrete actual being $B$, because $\mathfrak{D}$ is a set-theoretical representation of the qualitative results of experiments or observations extracted from some phenomenon that necessarily involves $B$; the embeddability relation between data structure $\mathfrak{D}$ and the model $\mathfrak{S} \in \boldsymbol{T}$ then acts as the simulacrum of the nexus between the abstract model (structure, theory) and the concrete actual being B. But this is not good enough. We don't want simulacra. We want the real thing. Come on.

We shall presently return to this Problem of the Lost Beings; for now we focus our attention on the data structures: for as soon as we have data structures, then, besides the Problem of the Lost Beings, no other problems will appear? Not quite, as we shall see in the next Section. ${ }^{23}$

\section{The Sea of Stories}

Suppes' larger programme in the philosophy of science encompasses Measurement Theory, which concerns the following. We begin by stating the obvious truth that all our observations are qualitative; our sensory organs are not pieces of measurement apparatus whose numerical results we somehow read off 'inside our minds'. The world we see around us seems to consist broadly speaking of mostly medium-sized dry material objects, moving slowly about or staying put. Science teaches us that everything we see is the result of electro-magnetic interaction between material objects and our eyes via a narrow window in the spectrum of all electro-magnetic radiation (400-800 nm), above a threshold intensity (below which our eyes do not register anything) and below some upper bound (above which our eyes burn). ${ }^{24}$ Now, a very specific kind of observations, obtained in certain contexts we call scientific experiments, we choose to represent numerically by using pieces of measurement apparatus, and we call these numerical representations data or measurements. Says Suppes [1960: 297]:

The maddeningly diverse and complex experience which constitutes an experiment is not the entity which is directly compared with a model of a theory. Drastic assumptions of all sorts are made in reducing the experimental experience, as I shall term it, to a simple entity ready for comparison with a model of the theory. 
That simple entity is the data structure. From the 1950ies onwards Suppes synthesised various approaches to the problem of under what conditions we are justified to represent qualitative observations quantitatively, i.e. numerically. This is what Measurement Theory is all about. ${ }^{25}$ For example, by first characterising the binary relation 'is heavier than' pertaining to qualitative observations on a pair of scales, and then proving that this relation can be represented mathematically by 'is larger than' on the real numbers, one is justified in introducing mass as a real-number-valued physical magnitude. Then it makes sense to say, for instance, that one mass is 3.74 as large as another. To contrast, one cannot characterise the binary relation 'is in love with' such that a similar representation theorem can be proved, which means there is no justification in saying that Charles Bovary loved Emma 3.74 as much as Rodolphe Boulanger and Léon Dupuis did. To utter these words is not to say anything; it is to utter nonsense.

For the sake of exposition, let us consider the numerical data set

$$
D(n, k) \subset\{1,2, \ldots, k\} \times \mathbb{Q}^{n},
$$

where $n, k \in \mathbb{N}$, which consists of $(n+1)$-tuples, $\left\langle j, q_{1}, q_{2}, \ldots, q_{n}\right\rangle$, with $j$ running from 1 to $k$, so that $k$ is the cardinality of $D(n, k)$. The data set $D(n, k)$, which is settheoretically a relation, forms the following data structure (data structures are always relation structures $\left.{ }^{26}\right)$ :

$$
\mathfrak{D}(n, k) \equiv\langle\mathbb{N}, \mathbb{Q}, D(n, k)\rangle \text {. }
$$

Logically speaking, every conceivable data set can be found in an actual experiment, but not every data set is found. Call the ones that have been found in some experiment or observation an actual data set. We now face two problems, which we address successively.

$1^{\circ}$. Which data sets of all possible ones are actual? When we want to express ourselves formally, there seems to be no other way than to accept in $\mathcal{L}_{\epsilon}$ 'actual' as a primitive monadic predicate of data sets. For suppose we try to provide a definition: a data set is actual iff it is obtained by performing some experiment or observation. The definiens stands as much in need of explication as the definiendum: the notion of 'a performed experiment' is as clear, or as obscure, as what an actual data set is. Etc. A simple pragmatic and informal yet defeasible sufficient condition for a data set to be actual is as follows: if a data set is published in some respectable scientific journal, then it is actual. Further, a data set is actual only if it involves an actual concrete being.

$2^{\circ}$. Suppose we have some actual data set. For which scientific theory is this data set supposed to be relevant?

Consider, for the sake of concreteness, a data set $D(2,10)$ of type $(9)-n=2$ and $k=10-$ and the following four simple physical experiments. 
(i) We measure the length $\left(l^{\prime}\right)$ of the image on a screen of a little wooden stick we hold in front of a positive lens outside its focal distance; we also measure the length $(l)$ of the stick itself; we have ten sticks. In this manner we obtain ten ordered triples of type $\left\langle j, l, l^{\prime}\right\rangle \in \mathbb{N} \times \mathbb{Q}^{2}$, which we collect in data set $D_{\text {lens }}(2,10)$.

(ii) We measure the direct current $(I)$ and the voltage $(U)$ over one resistor ten times, for different voltages; then we obtain ten triples of type $\langle j, I, U\rangle \in \mathbb{N} \times \mathbb{Q}^{2}$, which we collect in data set $D_{\text {elec }}(2,10)$.

(iii) We measure the traveled distance $(d)$ of a free falling coin in vacuum at ten points in time $(\tau)$ before it hits the ground; then we obtain ten triples of type $\langle j, \tau, d\rangle \in$ $\mathbb{N} \times \mathbb{Q}^{2}$, which we collect in data set $D_{\text {fall }}(2,10)$.

(iv) Finally, we measure the height of a mercury column $(h)$ at different instances during the day $(t)$; then we obtain ten triples of type $\langle j, t, h\rangle \in \mathbb{N} \times \mathbb{Q}^{2}$, which we collect in data set $D_{\mathrm{Hg}}(2,10)$.

Again, for which scientific theories are these data sets supposed to be relevant? Clearly the answer is: (i) $D_{\text {lens }}(2,10)$ is relevant for ray optics, (ii) $D_{\text {elec }}(2,10)$ for the theory of electrical circuits, (iii) $D_{\text {fall }}(2,10)$ for Galilei's theory of kinematics, but also for Newtonian particle mechanics, and (iv) $D_{\mathrm{Hg}}(2,10)$ for meteorological theories. The problem is that we cannot come to know this by staring at these data sets, because all four of them comprise ten ordered pairs of positive rational numbers. A story has to be told about how and in which scientific context the data sets are obtained. Thus all actual data structures float in a sea of stories, that need to be told in order to know which data are relevant for which theory. Without such stories we cannot even begin to address the relation between theory and observation.

Are there any essential ingredients every such story must have? We answer in the affirmative. Every story must tell us, first, which concrete actual beings the measurements pertain to, and, secondly, what is measured, which involves a specification of the units. Our presentation of the results of the four simple experiments (i)-(iv) above was in this sense incomplete: we should have added the relevant units. Notice however that providing units does not tell the complete story, because the ordered pairs in both (iii) $D_{\text {fall }}(2,10)$ and (iv) $D_{\mathrm{Hg}}(2,10)$ have units of time (second occupant) and length (third occupant). Confusing $D_{\text {fall }}(2,10)$ and $D_{\mathrm{Hg}}(2,10)$ would lead to a refutation of Newtonian particle mechanics and to doomsday-weather forecasts.

In telling the stories of how and in which contexts experimental results are obtained, we use language, we use words that express concepts and we use sentences that express propositions. When we arrive, by telling some story, with our data structure in the realm of theories, we know for which scientific theory or theories the data 
structure is relevant. But when we only have rigorous construals of all scientific theories in accordance with the S-View (7), to which of the sets of set-structures should we go in order to find a structure that embeds an obtained data structure? The 5 -View lacks the resources to tell the necessary stories: language. Call this the Problem of the Unavailable Stories. This problem leads us to the next Section, where we inquire into the conceptual and propositional content of the set-theoretical construals; it will give rise to our next critical reflection on the $\mathfrak{S}$-View.

\section{Concepts and Propositions at Bay}

We call to mind again that the epistemic aim of science is to provide knowledge of concrete actual beings; call theoretical scientific knowledge the kind of knowledge obtained by scientific inquiry that is stored in theories, and hence somehow in the models that constitute the theory according to the $\mathfrak{S}$-View (7). A necessary condition any model $\mathfrak{S}$ must pass in order to be considered as a piece of theoretical scientific knowledge about a particular kind of concrete actual being $B$ is that $\mathfrak{S}$ should embed all the relevant actual data structures obtained by observing B or performing experiments with B. But what does set-structure $\mathfrak{S}$, or a theory for that matter $(T \ni \mathfrak{S})$ say about $B$ ? Where has the conceptual and propositional content of the scientific theory $\mathbf{T}$, as used by scientists, gone to when reconstructed as $\boldsymbol{T}$ ? Call this the Problem of the Lost Content. ${ }^{27}$ Saying that force in classical mechanics is, first, a real 3-vector, $\mathbf{f} \in \mathbb{R}^{3}$, is saying something about the concept of force, namely that it has a direction in $\mathbb{R}^{3}$, which is its direction in 3-dimensional space when and only when we take $\mathbb{R}^{3}$ to represent 3-dimensional space, and, secondly, that force has a strength, or magnitude, when and only when we take length $\|\mathbf{f}\| \in \mathbb{R}^{+}$to represent this strength. But these explications go beyond the set-theoretical sentence ' $f \in \mathbb{R}^{3}$ ', because they involve the concepts of direction in 3dimensional space and strength, whose content clearly goes beyond the language of pure set-theory $\left(\mathcal{L}_{\in}\right.$, in which we can only talk about pure sets).

Furthermore, where and how does truth fit in the $\mathfrak{S}$-View? If there are 'truthmakers' of propositions that are somehow determined by means of $\mathfrak{S}$ (or of $\boldsymbol{T}$ ), then surely they are, or somehow reside in, the concrete actual being $B$ that $\mathfrak{S}$ (or $T$ ) is supposed to be about. For if $B$ is neither the truth-maker nor involved in the truth-making of sentences about $B$, then the concept of truth gets dissociated from the actual concrete beings, and such a concept of truth does not seem to be the the concept of truth that is used and understood in science. Surely in science truths and falsehoods are truths and falsehoods about the world. What else could they be about? Well, how does this precisely work, then, according to the $\mathfrak{S}$-View? 
Once again, the $\mathfrak{S}$-View passes over in silence, this time about the question what the relation is between the structures that constitute the theory and the beings the theory is supposed to be about (the Problem of the Lost Beings of Section 4 re-surfaces). Perhaps there is a profound wisdom in this silence. For what would count as possible answer to these questions?

Evidently, when we are asking of how this precisely works, we are asking for more words. We are asking for sentences that express propositions about being $B$, and these propositions surely involve concepts that express, presumably, properties of $B$ and relations between $B$ and other beings. Let model $\mathfrak{S} \in \boldsymbol{T}$ be supposed to be about being $B$. Those propositions and concepts expressed by the sentences and words that we are asking for will be the truly ontological ones, because they are 'directly' about B, about B an sich, as B really is, in and of itself, without any mediation by the products of science (model $\mathfrak{S}$, theory $T$ ). These propositions and concepts are expressed in what we, then, may call the ontological language of being $B\left(\mathcal{L}_{\text {ont }}\right)$. Next $\mathcal{L}_{\text {ont }}$ somehow has to be related to $\mathcal{L}_{\epsilon}$, in particular to the part of $\mathcal{L}_{\epsilon}$ that defines $\mathfrak{S}$ and $T(6)$, in order to establish the desired connexion between $\mathfrak{S}$ and $B$. Certainly we would like to have true sentences of $\mathcal{L}_{\text {ont }}$ about $B$; all ontological truths about $B$ may be said to express our ontological knowledge of $\mathrm{B}$. When $\psi(\mathrm{B}) \in \operatorname{SENT}\left(\mathcal{L}_{\text {ont }}\right)$ is a sentence is about $\mathrm{B}$ that is made true by $B$, and ' $=$ ' abbreviates this truth-maker relation, then what we are after is to fill in the dots in the following expression:

$$
B \models \psi(B) \text { iff } \ldots \ldots \ldots .\left(\text { in } \mathcal{L}_{\epsilon}\right) \text {. }
$$

But how are we going to find out whether $\psi(B)$ is true or false? By contemplation in the proverbial armchair? By thinking deeply and uncompromisingly about B? Or by combining reason with observation and imagination in that flabbergastingly successful manner that forms the beating heart of science? The second option surely seems promising. But recall that propositons and concepts expressed in $\mathcal{L}_{\text {ont }}$ were supposed to make sense of what science tells us about $B$. We were inquiring about the relation between $\mathfrak{S}$ and $B$. The 'ontological theory' we were after, in order to provide us with the desired ontological knowlegde of $B$, gives rise either to a doubtful programme of armchair science or to a scientific research project. In the last-mentioned case we are eating our tail. What went wrong?

Something went wrong in the beginning: we should not want to have $\mathcal{L}_{\text {ont }}$ in the first place, in order to talk about B. But is this because B as it really is, in and of itself, is epistemically inaccessible for us, as Kant would have it, so that the idea of considering $\mathcal{L}_{\text {ont }}$ is a senseless exercise? Or when B as it really is, in and of itself, somehow is epistemically accessible for us, do we not already have some part of the language of 
science to talk about $B$, namely the language of the scientific theory $T$ that is supposed to be literally about $B$, as Ampère countenanced? Is not $\mathbf{T}$ supposed to provide us with knowledge of concrete actual beings expressed by true and justified sentences in $\mathbf{T}$ ? If everything we want to know about $B$ is however not provided by $T$, then we must engage in more scientific research about $B$ in order to refine or to extend or to replace T. What else is there to do? And this lands us in our original problem: a set-structure $\mathfrak{S} \in T$ is defined in $\mathcal{L}_{\epsilon}$ and therefore cannot tell us anything about $B$ whilst $T$ is all that science will ever be able to produce on the current construal.

Perhaps we have to take a closer look at Suppes' arguments for his claim that his models $(\mathfrak{S} \in T)$ can be taken to be what working scientists call 'models'. What we have not mentioned so far is that Suppes was specifically inspired by Tarski's creation of Model Theory in Logic and that this has been the basis for Suppes' claim. ${ }^{28}$ Perhaps we find a clue here. Let's see.

Tarski's conception of a model is, properly construed and denoted, a triple

$$
\mathcal{M}=\langle\mathfrak{S}, R, \mid=\rangle,
$$

consisting of a set-structure $\mathfrak{S}$ living in the domain of meta-discourse ( $\mathbf{V}$ whenever the meta-theory is also some set-theory, which usually is the case), a Reference-map $R$ sending terms in a formal language $\mathcal{L}$ to items of $\mathfrak{S}$ and an inductively defined map $=$ from $\operatorname{SENT}(\mathcal{L})$ to the semantic values 'true' and 'false' (also known under various other names: satisfaction, truth-map, semantic valuation).

$$
\mathcal{M} \text { is a model of } \mathrm{T} \text { iff }\langle\mathfrak{S}, R\rangle \mid=\mathrm{T} \text {. }
$$

We have all learned this in our Logic courses. The relevant Suppesian model here is the set-structure $\mathfrak{S}$, not $\mathcal{M}(12)$. Suppes has argued that $\mathfrak{S}$ can be identified with what is called a 'model' by working scientists. So Suppes discards the semantics $(R$, $=)$; this is why the name 'semantic view' for Suppes' view is not just a misnomer but a terminological howler. But then what if we amend the $\mathfrak{S}$-View by taking the full triples $\mathcal{M}$ (12) aboard rather than only $\mathfrak{S}$ ? We then could take the set of all of them as the reconstruction of $\mathbf{T}$. The ensuing view of scientific theories could be truly called the Semantic View:

$$
\mathcal{T}=\left\{\mathcal{M}|\langle\mathfrak{S}, R\rangle| \mathrm{T} \wedge \mathfrak{S} \in \mathbf{V}_{\omega+g}\right\}
$$

to be combined with verified relevant observation sentences $\mathcal{O}_{t}(\mathbf{T})$ or actual relevant data structures $\mathcal{D}_{t}(\mathbf{T})$. Sentences and truth are now waiting just around the corner, ready to be amalgamated into the proper view of what a scientific theory is. 
This $\mathcal{T}(14)$ however does not seem to be what we want, because a Tarskian model $\mathcal{M}$ is a model of a formalised theory, of a set of sentences in a formal language, such as $\mathrm{T}$ (2) is. Of all admissible truth-maps the one is chosen that makes, in collaboration with a reference-map $R$ (from terms in the language to elements of $\mathbf{V}$ ), the axioms of $\mathrm{T}$ true. From $\mathcal{T}(14)$ we see that the Tarskian model presupposes part of the $\mathcal{L}$-View on theories (4). The set of all triples $\mathcal{M}(12)$ such that they are all models of a formal theory $\mathrm{T}$ is denoted as: $\operatorname{Mod}(T)$. Then $\mathcal{T} \subset \operatorname{Mod}(T)$, but if we re-define $\operatorname{Mod}(T)$ as to permit only structures in $\mathbf{V}_{\omega+g}$, as in (14), then

$$
\mathcal{T}=\operatorname{Mod}(\mathrm{T}) \text {. }
$$

In order to see $\operatorname{Mod}(T)$ as the proper reconstrual of scientific theory $T$, we first need $\mathrm{T}$. But what is $\mathrm{T}$ a formalisation of? If a formalisation of $\mathbf{T}$, then we are back in the L-View.

One way to avoid these problems is as follows. We return to the $\mathfrak{S}$-View (7) consider the extension $T$ (6) not as the ultimate but as the penultimate step towards a rigorous construal of scientific theory $\mathbf{T}$. In order to prepare for the ultimate step, we consider one of Van Fraassen's arguments in favour of his version of the semantic view (cf. reason VI at the end of Section 2):

In any tragedy, we suspect some crucial mistake was made at the very beginning. The mistake, I think, was to confuse a theory with the formulation of a theory in a particular language.

(...)

Words are like co-ordinates. If I present a theory in English, there is a transformation which produces an equivalent description in German. There are also transformations which produce distinct but equivalent English descriptions. This would be easiest to see if I were so conscientious as to present the theory in axiomatic form; for then it could be rewritten so that the body of theorems remains the same, but a different subset of those theorems is designated as the axioms, from all the rest follows. Translation is thus analogous to co-ordinate transformation - is there a co-ordinate-free format as well? The answer is Yes (although the banal point that I can describe it only in words obviously remains).

$(\ldots)$

Suppes' idea was simple: to present a theory, we define the class of models directly, without paying any attention to questions of axiomatisability in any special language, however relevant or logically interesting that might be. ${ }^{29}$

Van Fraassen draws a comparison with physical space-time theories and co-ordinate systems on space-time. For centuries such theories were formulated in terms of co- 
ordinates, which were taken to be labels of space-time points. In order to express that choosing a particular co-ordinate system is a pragmatic issue without any physical relevance, laws of nature formulated using a particular co-ordinate system were required to have the same 'physical content' when a co-ordinate transformation was performed on the laws. After Einstein had used Riemann's concept of a differentiable manifold in the formulation of his General Theory of Relativity, this mathematical structure was soon also used to re-formulate Einstein's Special Theory of Relativity (Minkowski space-time, Robb space-time) and Classical Electro-Dynamics (idem), Classical Mechanics (Galilean space-time, Newtonian space-time), without and with his theory of universal gravitation (Newton-Cartan space-time), In the language of Riemann's differential geometry, it has turned out to be possible to formulate laws of physics (that characterise a physical space-time theory) without mentioning co-ordinate systems, so as to make the physical irrelevance of co-ordinate systems manifest. Hence Van Fraassen compares the general framework of differential geometry and a differentiable manifold to the general framework of set-theory and a set-structure, respectively; then he compares the co-ordinate-free formulation of a physical space-time theory with the language-free formulation of a scientific theory $\mathbf{T}$ through a set $\mathbf{T}$ of set-structures $\mathfrak{S}$ (6). The banal point is that we use $\mathcal{L}_{\epsilon}$ to define $\mathfrak{S}$ and $T$. So $\mathcal{L}_{\epsilon}$ has nothing to do with the language used by scientists in text books and review articles to characterise $\mathbf{T}$ but is a mere means to characterise $\mathfrak{S}$ and $\boldsymbol{T}$. We then further compare a co-ordinate system on space-time with a formulation of $\mathrm{T}$, both chosen for the sake of convenience, and transformations between co-ordinate systems with translations between formulations of $\mathbf{T} .^{30}$

All this looks pretty appealing, especially when displayed:

$\begin{array}{lll}\begin{array}{l}\text { differential geometry } \\ \text { differentiable manifold }\end{array} & \text { set-theory } \\ \begin{array}{l}\text { co-ordinate-free formulation } \\ \text { of a space-time theory } \\ \text { co-ordinatisation of a space-time }\end{array} \leadsto \begin{array}{l}\text { set-structure } \\ \text { language-free formulation } \\ \text { of a scientific theory }\end{array} \\ \begin{array}{l}\text { atlas of all co-ordinate transformations } \\ \text { formulation of a scientific theory }\end{array} & \text { all formulations of a scientific theory } \\ \text { co-ordinate transformations } & \text { formulation translations }\end{array}$

There is, however, a problem.

When we formulate $\mathbf{T}$ rigorously - or "conscientiously", as Van Fraassen puts it in the quotation displayed above - as a formal-deductive system $\mathrm{T}$ (2), we obtain a lexicon of a particular signature, which tells us how many names, how many sorts of 
variables and how many primitive predicates of every adicity there are. This will fix the signature (of the structure 5 ) of the models of $T(12)$, that is, of all the models in $\operatorname{Mod}(T)$, or so it seems. A model having a different signature cannot, then, be a model of $T$ ? If we translate $\mathcal{L}$ into another language, say $\mathcal{L}^{\prime}$ (every item of the lexicon of $\mathcal{L}$ is translated in terms of the lexicon of $\mathcal{L}^{\prime}$ ), and choose axioms in $\mathcal{L}^{\prime}$ whose deductive closure yields the formal theory $\mathrm{T}^{\prime}$, such that all translated axioms of $\mathrm{T}$ become theorems of $\mathrm{T}^{\prime}$, and conversely, then "the body of theorems remains the same" (Van Fraassen, above): $T=T^{\prime}$. But since in general the signature of $\mathcal{L}^{\prime}$ is different from that of $\mathcal{L}$, the models of $\mathrm{T}^{\prime}$ and of $\mathrm{T}$ will be distinct, so the classes of their models are disjoint:

$$
\operatorname{Mod}(\mathrm{T}) \cap \operatorname{Mod}\left(\mathrm{T}^{\prime}\right)=\varnothing
$$

Yet (16) seems incompatible with $T=T^{\prime}$, for if $T=T^{\prime}$, then $\operatorname{Mod}(T)=\operatorname{Mod}\left(T^{\prime}\right)$. Right? Wrong. On the basis of the L-View (4), it does not follow from $T=\mathrm{T}^{\prime}$ that the theories are identical because to draw that conclusion the lexicons have to coincide too. For the $\mathcal{L}$-View, the formulation counts. Since the lexicons of $\mathcal{L}$ and $\mathcal{L}^{\prime}$ are different, the signatures of the models of $T$ and $T^{\prime}$ are different, so that indeed $\operatorname{Mod}(T) \cap$ $\operatorname{Mod}\left(T^{\prime}\right)=\varnothing$. A more explicit notation such as ${ }^{\prime} \operatorname{Mod}(T, \operatorname{LEX}(\mathcal{L}))^{\prime}$ would have prevented the fallacious inference from $T=T^{\prime}$ to $\operatorname{Mod}(T, \operatorname{LEX}(\mathcal{L}))=\operatorname{Mod}\left(T^{\prime}, \operatorname{LEX}\left(\mathcal{L}^{\prime}\right)\right)$.

But now notice that there is a straightforward manner to amend the $\mathcal{L}$-View slightly so as to meet Van Fraassen's challenge to provide language-free characterisation of scientific theory $\mathbf{T}$. We could simply universally quantify away the lexicon from (4) and drop uno icto the distinction between 'observation' and 'theoretical' predicates. Specifically, we identify a theory as a class of all and only inter-translatable formal theories; loosely and symbolically:

$$
\begin{aligned}
\mathcal{A}_{\mathrm{T}} \equiv\left\{\left\langle\mathrm{T}^{\prime}, \operatorname{LEX}\left(\mathcal{L}^{\prime}\right)\right\rangle \mid\right. & \exists g: \operatorname{LEX}\left(\mathcal{L}_{\mathrm{T}}\right) \rightarrow \operatorname{LEX}\left(\mathcal{L}^{\prime}\right): g[\mathrm{~T}]=\mathrm{T}^{\prime} \text { and } \\
& \left.\exists g^{\operatorname{inv}}: \operatorname{LEX}\left(\mathcal{L}^{\prime}\right) \rightarrow \operatorname{LEX}\left(\mathcal{L}_{\mathbf{T}}\right): g^{\operatorname{inv}}\left[\mathrm{T}^{\prime}\right]=\mathrm{T}\right\},
\end{aligned}
$$

where bijective map $g$ is a translation between the lexicons. Call now this the FormulationIndependent Linguistic View, or [L]-View for brevity:

$$
\left\langle\mathcal{A}_{\mathrm{T}}, \vdash, \mathcal{O}_{t}(\mathbf{T})\right\rangle
$$

where ' $\mathcal{A}_{\mathrm{T}}$ ' alludes to 'atlas'. An immediate consequence of (17) is that one member of this class fixes the models of all its other members.

So the bodies of all formal theories in this class coincide; notice that it is an equivalenceclass because the relation 'is inter-translatable to' is an equivalence-relation; every $\mathcal{A}_{\mathrm{T}}$ is a member of the quotient-class of the class of all elementary formal theories divided 
by the relation of inter-translatability. Then what the theory says about the world, i.e. its propositional content, expressed in $\mathrm{T}$, is invariant under translations. Hence our characterisation (18) of $\mathbf{T}$ is, although not language-free, independent of any specific formulation. The conceptual content of the theory is formulation-dependent and hence language-dependent: it is expressed by a lexicon and lexicons change under translation.

Now, to provide a formulation-free rigorous characterisation of $T$, e.g. as in $T$ (6) of the $\mathfrak{S}$-View, is one way to make a rigorous characterisation formulation-independent (as analogous to a co-ordinate-independent formulation of a physical space-time theory), yet it is not the only way. Another way is to quantify the formulation-dependence universally away, as we just have done, and make the propositional content of a theory independent of its formulation, i.e. of its lexicon.

For those who insist on a language-free charactersation of $\mathbf{T}$ nonetheless, rather than a formulation-independent one, we point out that also in a co-ordinate-free formulation of physical space-time theories in terms of differentiable manifolds, the co-ordinate systems are not eradicated at all. Physical space-time theories are not free of co-ordinate systems. On the contrary, the structure of a differentiable manifold involves by definition an atlas, which is the set of all local co-ordinate systems on the open cover of the space-time continuum; and it is in terms of these co-ordinate systems and their transformations that the differentiability of the manifold is characterised. There is no such thing as a co-ordinate-free differentiable manifold. To a certain extent the co-ordinatefree formulation of physical laws in space-times theories is therefore a fata morgana, because when these laws are explicated, including an explication of the concepts used in the formulation of these laws, all co-ordinate systems will inevitably re-appear on the stage. The independence of physical laws from co-ordinate systems used to be not manifest because co-ordinates were mentioned in the formulation of physical laws; this was guaranteed by imposing a co-variance requirement on physical laws under co-ordinate transformations. In the $[\mathcal{L}]-V i e w(18)$ something exactly analogous happens.

The formulation-independence is manifest in the language-free $\mathfrak{S}$-View (7). We are now prepared to set the promised ultimate step in order to solve the Problem of the Lost Content in the $\mathfrak{S}$-View. Set $T$ consists of structures $\mathfrak{S}(6)$. When part of a Tarskian model $\mathcal{M}(12)$, structure $\mathfrak{S}$ fixes the signature of $\mathcal{M}$. This fixes in turn the signature of a formal language, call it $\mathcal{L}_{\mathfrak{S}}$, in which we can formulate a theory that has $\mathcal{M}$ as its Tarskian model; call such a theory $\mathrm{Th}(\mathfrak{S})$. We can consider the class of exactly the theories of $\mathfrak{S}$ :

$$
\boldsymbol{K}_{\mathfrak{S}} \equiv\left\{\left\langle\operatorname{Th}(\mathfrak{S}), \operatorname{LEx}\left(\mathcal{L}_{\mathfrak{S}}\right)\right\rangle|\exists R:\langle\mathfrak{S}, R\rangle| \operatorname{Th}(\mathfrak{S}) \text { and } \mathfrak{S} \in \mathbf{V}_{\omega+g}\right\} .
$$


Do the theories in $K_{\mathfrak{S}}$ have the same signature, namely the one of $\mathfrak{S}$ ? Closer inspection reveals they do not, because every translation of member $\left\langle\operatorname{Th}, \operatorname{LEX}\left(\mathcal{L}_{\mathfrak{S}}\right)\right\rangle$ will also be made true by $\langle\mathfrak{S}, R\rangle$. Every structure $\mathfrak{S} \in T$ now comes with a class $K_{\mathfrak{S}}$ of theories, having various lexicons, all of which are made true by $\langle\mathfrak{S}, R\rangle$. The following ordered pair should then be an improvement on the $\mathfrak{S}$-View (7) as the construal of what working scientists call a 'model':

$$
\left\langle\mathfrak{S}, K_{\mathfrak{S}}\right\rangle \text {. }
$$

Roughly, a model is a structure and all its formulations. When we define the propositional content of $\mathfrak{S}$ as all inter-translatable formulations, then model (20) is a structure and its propositional content. ${ }^{31}$ Let $\boldsymbol{T}_{\tau}$ now be the class of all such ordered pairs, where $\mathfrak{S}$ meets the antecedently defined Suppes predicate $\tau(\cdot)$ in $\mathcal{L}_{\epsilon}$ :

$$
T_{\tau} \equiv\left\{\left\langle\mathfrak{S}, K_{\mathfrak{S}}\right\rangle \mid \tau(\mathfrak{S})\right\}
$$

Then our improved rigorous construal of $\mathbf{T}$ is

$$
\left\langle T_{\tau}, \mathcal{D}_{t}(\mathbf{T})\right\rangle
$$

Let us call this the Structural View, or briefly the $\boldsymbol{K}_{\mathfrak{S}}$-View.

The $K_{\mathfrak{S}}$-View (22) is an improvement, because now we have lots of languages and theories of $\mathfrak{S}$ - formulations of theories if you like - which we can appeal to for locating the conceptual and propositional content of $\mathbf{T}$ : we now have sentences that can be true or false; and we have the predicates of the lexicons to express concepts. Choice of language does not matter for the propositional content of $\boldsymbol{T}_{\tau}(21)$, as choice of co-ordinate system does not matter for the physical content of space-time theories, because all sets $\operatorname{Th}(\mathfrak{S})$ of sentences generated by $\mathfrak{S}$, and made true by Tarskian model $\mathcal{M}(12)$ that has $\mathfrak{S}$ as its structure, can have wildly differing lexicons and these are all included in $\boldsymbol{T}_{\tau}$ (21). This solves the Problem of the Lost Content. Further, it has all the resources to solve the Problem of the Unavailable Stories.

To recapitulate, we have begun this Section by raising the difficulty where the conceptual and propositional content of scientific theory $\mathbf{T}$ has gone to when construed in accordance to the S-View (7), namely as a set of set-structures defined in the language of pure set-theory $\left(\mathcal{L}_{\epsilon}\right)$. This Problem of the Lost Content is related to the Problem of the Lost Beings we raised earlier when we wondered where the $\mathfrak{S}$-View allocates the concrete actual beings (B). For $\mathbf{T}$ is supposed to provide us with scientific knowledge, in the sense of scientifically justified true propositions about concrete actual beings. We briefly explored the possibility of an ontological language $\left(\mathcal{L}_{\text {ont }}\right)$ to talk directly about the beings (so as to solve both of the mentioned problems uno icto), but we soon 
discovered that we were eating our tail. Then we moved, inspired by Suppes' inspiration, from the Suppesian model $\mathfrak{S}$ to the Tarskian model $\mathcal{M}$ (12). Notwithstanding the fact that Tarskian models gave us languages to talk about abstract set-structures rather than about the concrete actual beings (B) that $\mathbf{T}$ is about, we proceeded, inspired by Van Fraassen's analogy with co-ordinate-free characterisations of physical space-time theories, to loosen the bond between structure (ㄷ) and formulation (notably the lexicon of $\mathcal{L}$ ) by incorporating translations into the $\mathcal{L}$-View. This has led to the $[\mathcal{L}]$-View $(18)$, according to which $\mathbf{T}$ is the set of all formal formulations of $\mathbf{T}$. Then we re-introduced formulations of theories of structures into the $\mathfrak{S}-V i e w$, but in such a manner that all formulations are incorporated that leave the propositional content of the theory invariant. This led to the $K_{\mathfrak{S}}$-View (22), which solves the Problems of the Lost Beings, of the Lost Content and of the Unavailable Stories.

Should we now choose the $[\mathcal{L}]$-View (18) or the $K_{\mathfrak{S}}$-View (22) as the best reconstrual of scientific theories as they occur in science? In the next Section we answer this question.

\section{Towards Completion}

Einstein once said that we should make our scientific theories simple but not simpler. Mutatis mutandis for our views on scientific theories. Consider the two new views:

$$
\begin{aligned}
& {[\mathcal{L}] \text {-View }:\left\langle\mathcal{A}_{\mathbf{T}}, \mathcal{O}_{t}(\mathbf{T})\right\rangle,} \\
& \boldsymbol{K}_{\mathfrak{S} \text {-View }}:\left\langle\boldsymbol{T}_{\tau}, \mathcal{D}_{t}(\mathbf{T})\right\rangle,
\end{aligned}
$$

which are both markedly different from their progenitors, the $\mathcal{L}$-View (4) and the $\mathfrak{S}$ View (7), respectively. Both new Views look baroque, but the $[\mathcal{L}]$-View does not suffer from the formulation-dependence that crippled its predecessor, the $\mathcal{L}$-View (4), and the Structural View; further, it suffers neither from the Problem of the Lost Content nor from the Problem of the Lost Beings nor from the Problem of the Unavailability of the Stories - it does suffer from other problems, that lead to the decline of the L-View (see Section 2). The $\boldsymbol{K}_{\mathfrak{S}}$-View (23) also does not fall prey to the mentioned three Problems.

What should not come as a surprise is that on top of this, the gap between these two improved views (23) is not as wide as between the $\mathcal{L}$-View (4) and the S-View (7). For from (17) and (19) we deduce:

$$
\mathcal{A}_{\mathrm{T}}=K_{\mathfrak{S}}
$$

Logically speaking, both new views really are two sides of the same coin. Philosophically speaking, the gap remains sufficiently wide to prefer the $\boldsymbol{K}_{\mathfrak{S}}$-View (22) over the 
$[\mathcal{L}]$-View (18), because the starting point for this construal remains a set-theoretical predicate $\tau(\cdot)$ defining a type of set-structure $\mathfrak{S}$ (the Suppesian model keeps playing the main part), and because the formal theories and their inter-translations in $\boldsymbol{K}_{\mathfrak{S}}$ need never be spelled out (the formalising labour that is mandatory in the $\mathcal{L}$-View need not be performed). In the old $\mathfrak{S}$-View (7) the formulations are simply not there, which is why the conceptual and propositional content of $\mathbf{T}$ is lost and $\mathbf{T}$ floats helplessly around in the sea of stories; in the $K_{\mathfrak{S}}$-View (23) they are lying there for the taking and are officially acknowledged to be part of the full reconstrual of $\mathbf{T}$. Conceptual and propositional content can be hauled in at will and by law. Those propositions can and should be taken as being literally about the concrete actual beings, in exactly the same way as the proposition expressed by the Latin sentence 'Sol lucet' is about the Sun: we have dived deeply for the beings and here they are.

This does not mean that we have solved the problem of how precisely the structure $\mathfrak{S}$ relates to the concrete actual being $\mathrm{B}$, but it does mean that we have transformed the problem so as to subsume the transformed problem under one of the major problems of philosophy of the past century: the profound problem of how to understand the relation between the word and the world. Figure 2 depicts the situation we have arrived at.

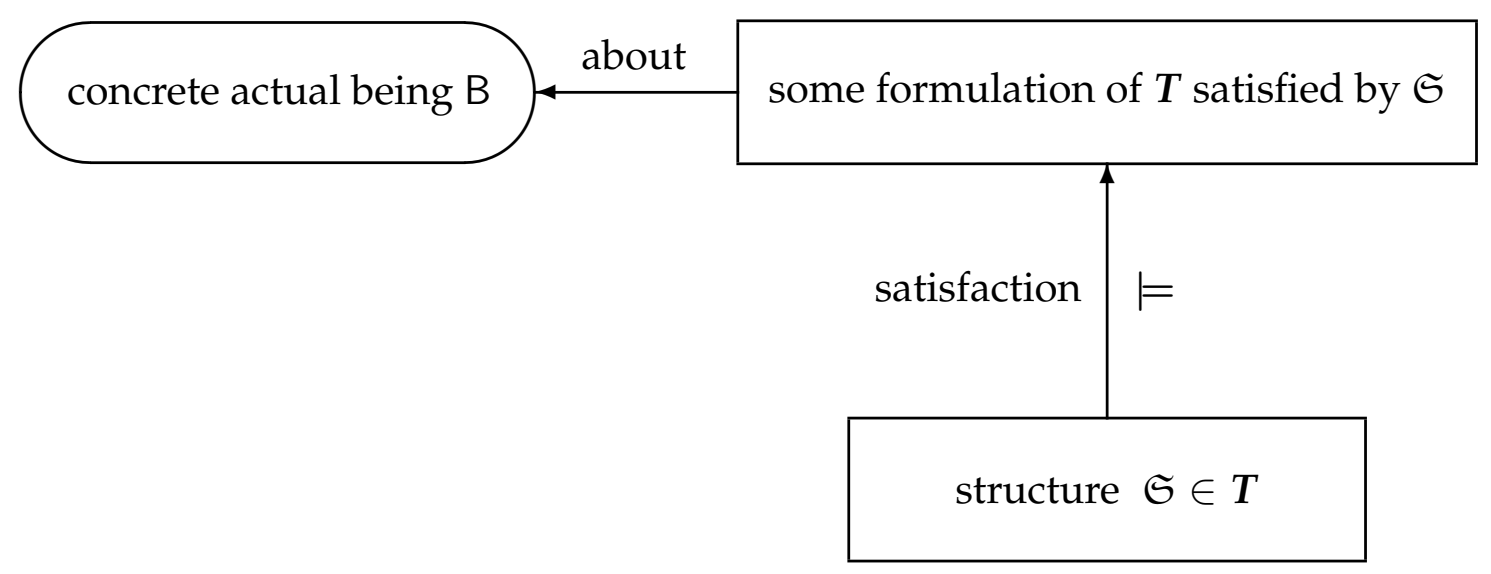

Figure 2: Beings in the $\boldsymbol{K}_{\mathfrak{S}}$-View (22)

About this profound problem, the $\boldsymbol{K}_{\mathfrak{S}}$-View (22) has little to say — we shall say a bit about it in the next and final Section. Yet at soon as it is granted that the $K_{\mathfrak{S}^{-}}$ View on scientific theories need not solve this profound philosophical problem - as for example propounders of the informal-structural view French and Ladyman [1999: 
115] hold - we can conclude, succinctly, that the $\boldsymbol{K}_{\mathfrak{S}}$-View (22) completes the Model Revolution at Stanford.

A complementary way to complete the Model Revolution, not to solve the problems we have tried to solve, but to solve other problems concerning experimental science rigorously, is outlined by P. Suppes (in this issue of Synthese).

\section{Exitum: Scientific Representation}

The concept of representation occurs in art, science, engineering, mathematics, astrology, logic, linguistics, cinematography, religion and literature, poetry included. Raising the question whether it is possible to have a substantive philosophical account of representation that covers all these varieties of human activity will cause mostly sceptical frowns rather than encouraging cheers. Did anyone ever conceive of this to be possible? The best way to proceed, then, seems to follow Wittgenstein's way: analyse how the word 'representation' is used in all these different contexts; then try to find out whether these uses have anything in common so as to identify that as the core of the concept of representation; and then, finally, to adopt this core as the characterisation or even definition of the concept of representation; if all this fails, we must presumably conclude that representation is a family-resemblance concept.

Our very first step is to restrict our focus on representation in science, that is, on scientific representation, and leave representation in all other mentioned fields aside, and further focus on scientific representation according to the $K_{\mathfrak{S}}$-View (22), that is, on structural scientific representation, or simply structural representation for brevity. ${ }^{32}$

What the $\boldsymbol{K}_{\mathfrak{S}}$-View straightforwardly seems to say about representation is depicted in Figure 3. 


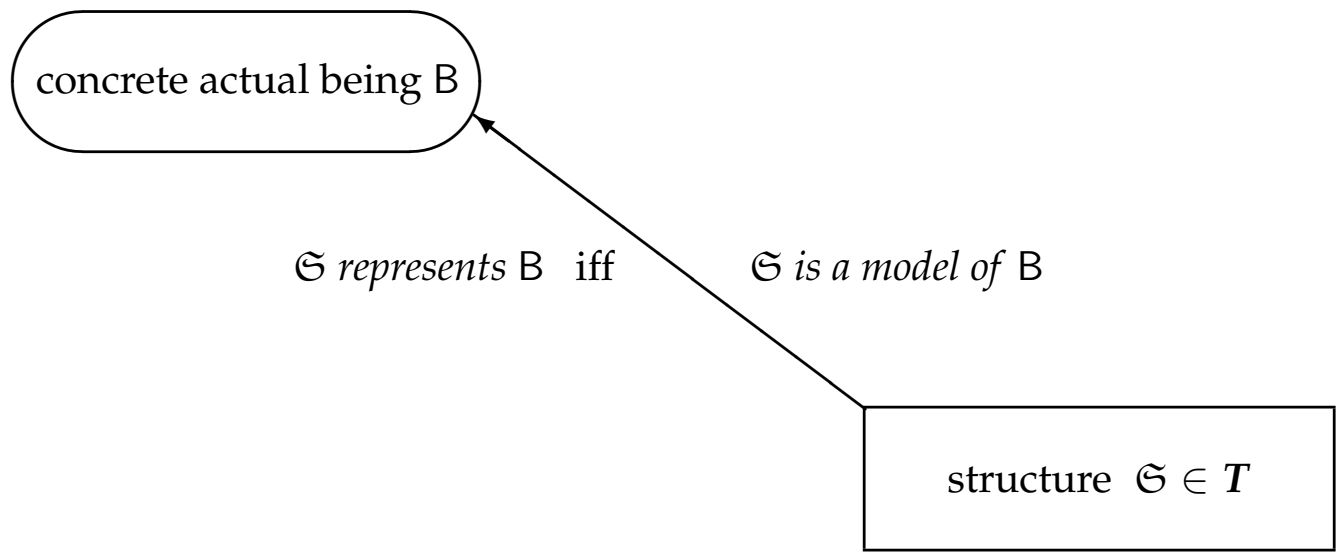

Figure 3: Representation according to the $\boldsymbol{K}_{\mathfrak{S}}$-View (22)

So 'representation' is another word for 'model'. R.N. Giere however argues that in order to make sense of (the practice of) science, representation should not be expressed by a dyadic predicate, but by a quadratic predicate, which reads in our terminology: ${ }^{33}$

scientist $S$ uses model $\mathfrak{S}$ to represent being $\mathrm{B}$ for purpose $P$.

Of course analysis (25) does not help a whiff to elucidate the relation between the representing structure $\mathfrak{S}$ and the represented being $B-$ on the contrary, (25) only complicates it. Furthermore, an analysis of the form (25) puts an end to what M. Suárez has called a naturalised analysis of representation, because the epistemic agents and their actions and purposes enter the analysis. Representation then has become partly a pragmatic concept. To represent is a manifestation of human agency.

R. Frigg, who also takes models to be the representing entities (see Figure 3), has listed six problems that a substantive philosophical account of scientific representation must solve: ${ }^{34}$

(F1) Ontological Problem. What sort of enties are models?

(F2) Clarification Problem. In virtue of what does a model represent something else?

(F3) Taxonomic Problem. What is the taxonomy of the various kinds of models that are used in science?

(F4) Demarcation Problem. How to distinguish scientific from non-scientific models? 
(F5) Epistemological Problem. How to acquire knowledge of the world (of the concrete actual beings) from the models?

(F6) Problem of Misrepresentation. How to account for the fact that refuted, erroneous, idealised, approximate, inaccurate models never fail entirely to represent?

By construing the representation-relation between structure $\mathfrak{S}$ and being $B$ as an isomorphism (or a sibling notion), Frigg has argued that the S-View (7) is unable to solve most of these problems. Although a thorough inquiry of whether the improved $K_{\mathfrak{S}^{-V i e w ~}}$ (22) fares better in meeting the Frigg Conditions (F1-F6) is beyond the scope of the current paper, a few quick answers seem in order.

(F1) Ontological Problem. Models are set-structures.

(F2) Clarification Problem.

(F3) Taxonomic Problem. No stumbling blocks in the $\boldsymbol{K}_{\mathfrak{S}}$-View for erecting some taxonomy of models used in science.

(F4) Demarcation Problem. Any set-structure in $\mathbf{V}_{\omega+g}$ can in principle be a model. The problem of what distinguishes the activity of science from other human activities wherein representation occurs, such as art, engineering, astrology, literature and cinematography, cannot and should not be solved by focusing on models only.

(F5) Epistemological Problem. There is lots of propositional content stored in $\boldsymbol{K}_{\mathfrak{S}}$ (19) in $\boldsymbol{T}_{\tau}$ (21) of the $\boldsymbol{K}_{\mathfrak{S}}$-View (22): there our scientific knowledge of the world is expressed.

(F6) Problem of Misrepresentation. When we choose tailor-made morphisms on a case by case basis, misrepresentation can easily be accounted for. Representor and represented have at least one resemblance (otherwise one would never be called a representation of the other), and precludes total misrepresentation.

M. Suárez [2003] has argued similarly that there are five arguments against a construal of the representation-relation as isomorphism (or a sibling notion).

(S1) Variety Argument. There are lots of entities that represent but are not setstructures.

(S2) Logical Argument (originally due to N. Goodman). The representation-relation has different properties than the isomorphism-relation: the aforementioned is neither reflexive nor symmetric nor transitive whereas the last-mentioned is an equivalencerelation. 
(S3) Misrepresentation Argument. A model can be mistargeted or can be idealised, approximate, inaccurate without loosing its representation-relation (cf. F6).

(S4) Non-necessity Argument. Representation-relations are not always isomorphisms (or sibling notions).

(S5) Non-sufficiency Argument. Isomorphisms (and sibling notions) are not always representation-relations.

Again, although a thorough inquiry of whether the improved $\boldsymbol{K}_{\mathfrak{S}}$-View (22) also falls prey to the Suárez Arguments is beyond the scope of the current paper, a few quick responses seem in order.

(S1) Variety Argument. We decide ab initio to deal with scientific representations only, which are (nearly) always set-structures.

(S2) Logical Argument. When we choose tailor-made morphisms on a case by case basis, this argument breaks down.

(S3) Misrepresentation Argument. When we choose tailor-made morphisms on a case by case basis, misrepresentation can easily be accounted for.

(S4) Non-necessity Argument. Suárez's [2003: 235-236] counter-examples from art can be set aside as irrelevant for representation in science.

(S5) Non-sufficiency Argument. Suárez's [2003: 236] argument that morphisms lack 'directionality', which is an essential feature of the representation-relation, cannot be met without extending the concept of representation to a form like (25).

I am under no illusion that these quick responses settle the representation debate as far as the $\boldsymbol{K}_{\mathfrak{S}}$-View (22) is concerned. At best they point in directions worth inquiring into. As Frigg [2006, §5] has emphasised, to construe the relation between a model $\mathfrak{S}$ and a being $B$ as a morphism of any kind presupposes that $B$ is a structure, because morphisms of any kind are defined as inter-structural relations. This ontological presupposition is often taken for granted, but stands, of course, also in need of inquiry - an inquiry that lies, too, beyond the scope of the current paper. ${ }^{35}$ When we avoid this presupposition by taking the representation-relation (or equivalently the modelof-relation; see Figure 3) as primitive, almost all of Suárez's arguments loose their force. But then Frigg's Clarification Problem (F2) remains unsolved.

One way to solve it is to adopt a truth-conditonal view of meaning. We then attempt to specify truth-conditions for the proposition: structure $\mathfrak{S}$ represents being $\mathrm{B}$ 
$(\boldsymbol{\Delta})$. When we take B to be some phenomenon, the truth-condition is in terms of the embeddability of the data structures extracted from B into $\mathfrak{S}$. For an empiricist, this is sufficient. For a realist, this is not sufficient. But then the truth-condition for $(\mathbf{\Delta})$, in particular when $B$ is unobservable, launches us on the battle ground of the realism debate. Alas! Beyond the scope of the current paper.

I have little to recommend my opinions. They come from one who desires honours, distinctions, and emoluments, but little; and who expects them not at all; who has no contempt for fame and no fear of obloquy; who shuns contention, though he will hazard an opinion; from one who wishes to preserve consistency; but who would preserve consistency by varying his means to secure the unity of his end; and, when the equipoise of the vessel in which he sails may be endangered by overloading it upon one side, is desirous of carrying the small weight of his reasons to that which may preserve his equipoise.

FINIS 
Acknowledgments. Many thanks to Roman Frigg (London School of Economics), who is responsible for the presence of a Section on representation, to Th. Kuipers (Groningen University), to Mauricio Suárez (Complutense Madrid) and to Bas van Fraassen (Princeton University) for remarks, and to Patrick Suppes (Stanford University) for a long responsive letter on an earlier version.

Affiliations. Faculty of Philosophy, Erasmus University Rotterdam, Burg. Oudlaan 50, H5-16, 3062 PA Rotterdam, E-mail: f.a.muller@fwb.eur.nl; and: Institute for the History and Foundations of Science, Dept. of Physics \& Astronomy, Utrecht University, Budapestlaan 6,WG-3.08, Utrecht, E-mail: f.a.muller@phys.uu.nl, The Netherlands

\section{Notes}

${ }^{1}$ See O'Brien [1967: 42-43].

${ }^{2}$ McKinsey, Sugar and Suppes [1953], McKinsey and Suppes [1953], [1955], Suppes [1954].

${ }^{3}$ For the canonical review of the Logical- Positivistic views on scientific theories, see Suppe [1974].

${ }^{4}$ Here it is admittedly taken for granted that we can identify a scientific theory in scientific writings; although this is not immune from philosophical doubt, it is hardly a speculative supposition for a science as, say, physics.

${ }^{5}$ At the meta-level we have some weak set-theory to make this all possible rigorously. The concept of 'object' here is the metaphysically thin notion as used by Frege and Quine: it can be anything over which we can quantify and for which we then must supply identity conditions - physical objects, persons, processes, events, mental states, space-time points, dreams, numbers, sets, actions, etc.

${ }^{6}$ By 'formalisation' we mean what Frege meant: spelling out the alphabet, and all the formation and deduction rules.

${ }^{7}$ Mathematical theories are about (particular kinds of) abstract objects only (numbers, geometrical figures, sets, structures); scientific theories are about concrete actual beings. The existence of the objects of mathematics is philosophically controversial; the existence of the objects of science is not, with the exception of the unobservable ones.

${ }^{8}$ This concept of observational adequacy should not be confused with Van Fraassen's well-known concept of empirical adequacy. Three differences between the concepts of observational adequacy (ObsAdeq) and empirical adequacy (EmpAdeq): (i) ObsAdeq relies on the distinction between theoretical and observational concepts whereas EmpAdeq does not rely on that; (ii) ObsAdeq depends on historical time whereas EmpAdeq is timeless (quantifies universally over historical time); (iii) EmpAdeq requires for its definition a conception of a scientific theory that results from the Model Revolution, because it cannot be defined in the conception we are expounding here. This last point, although explicitly and clearly stated in The Scientific Image (Fraassen [1980: 54-55]), has been poorly appreciated by the community of philosophers of science: witness that such eminent philosophers of science as M. Friedman, A. 
Musgrave and the late P. Lipton are among the poor appreciators — see Muller \& Van Fraassen [2008]. Both ObsAdeq and EmpAdeq rely on the distinction between observable and unobservable objects.

${ }^{9}$ Science harbours other kinds of knowledge as well, such as experimental results, knowledge of how to design and to conduct a scientific experiment, and of how to apply theories.

${ }^{10}$ See Suppe [1974: 50-51].

${ }^{11}$ See Da Costa \& Chuaqui [1988] for the definition of a 'Suppes-predicate'; for a friendly definition of Bourbaki's related concept of a set-structure, see Muller [1998: 106-115].

${ }^{12}$ Some morphism, that is, isomorphism, partomorphism, monomorphism, epimorphism, partial isomorphism and perhaps more; see Muller [1998: 125-126].

${ }^{13}$ This means one can iterate the power-set operation on $\mathbb{N}$, which we identify with $\omega$, googol $(g)$ number of times. This yields all the Cartesian product-sets to have all the mathematical objects that science needs and ever will need.

${ }^{14}$ Suppes [1974], Luce in Bogdan [1979: 93-110].

${ }^{15}$ See e.g. Putnam [1962], Toulmin [1974].

${ }^{16}$ Fraassen [1989: 225].

${ }^{17}$ Suppes [1954], [1968].

${ }^{18}$ See Suppes [1961], [1993], [2002], Bogdan [1979], Sneed [1971], Stegmüller [1976], Rantala [1978], Balzer, Sneed and Moulines [1987], Giere [1988], Costa \& French [1990], Muller [1998].

${ }^{19}$ Typical is Morgan \& Morrison [1999], who champion this approach with much clamor and less clarity; see Giere [1988] for less clamor and more clarity.

${ }^{20}$ Somewhat devious is to ignore the mathematics of $\mathbf{T}$ also in the $\mathcal{L}$-View and to take, by default, always $Z C$ as the mathematical part of every $\mathbf{T}$. Then $\operatorname{MATH}\left(\mathcal{L}_{\mathbf{T}}\right)=\{\in\}$ in (5).

${ }^{21}$ E.W. Beth [1968: 31-32]; this is the 2nd and revised edition of the first one from 1957.

${ }^{22}$ The correspondence between Beth's characterisation [1968: 31-32], where the conditions are labeled by Roman numerals, and De Jong \& Betti's [2008], is as follows: $\alpha-\mathrm{I}, \beta-\mathrm{IV}(\mathrm{b}), \gamma-\mathrm{III}, \delta$-II. Beth's IV(a) is the meaning of the fundamental concepts being evidently clear; his $\mathrm{V}(\mathrm{a})$ is that the principles should be obviously true; and his $\mathrm{V}(\mathrm{b})$ is the soundness of the deduction-relation, which is construed epistemically by De Jong \& Betti, as in condition $\zeta$.

${ }^{23}$ The essential content of Section 5, i.e. expounding the Problem of the Unavailable Stories, is at least ten years old: see Muller [1998: 284-292].

${ }^{24}$ For a scientific criterion of observability, see Muller [2005: §4,5].

${ }^{25}$ D. Luce in Bogdan [1979: 93]: "More than any other living person, Suppes has affected contemporary presentations of theories of measurement." For a review of Measurement Theory, see Humphreys [1994: 219-245]. Díez [1997] puts Suppes' role in historical perspective. For an elementary introduction to the subject, see Carnap [1966: 62-104]. Krantz et al. [1971] is one volume of the encylopedia-type series of books containing all data structures in science, together with representation and invariance theorems.

${ }^{26}$ Krantz et al. [1971], Humphreys [1994: 219-245].

${ }^{27}$ The $\mathcal{L}$-View is plagued by a similar problem, because its theoretical vocabulary is only partially interpreted, i.e. connected to observation predicates in the correspondence postulates of $T$ (2); the theoretical vocabulary remains underdetermined.

${ }^{28}$ Suppes [2002, 20-21]. Suppes [1960: 289]: "I claim that the concept of a model in the sense of Tarski may be used without distortion and as a fundamental concept ..."

${ }^{29}$ Fraassen [1989: 221, 5-6]. 
${ }^{30}$ Translation in Logic is a concept also due to Tarski; see Muller [1998: 170-173] for details. To formalise making a model of some theory is to define a translation from the object-language in the metalanguage.

${ }^{31}$ This idea was first proposed in Muller [2005: §6].

${ }^{32}$ Then we can gloss over examples and counter-examples of representation drawn from art etc. that M. Suárez [2003] mobilises against structural scientific representation.

${ }^{33}$ Giere [2003: 743]. Giere identifies 'general principles' that scientists employ to construct models of "features of the world" (of beings), and has no use any longer for the concept of a theory [2004: 744-747]. But a precise characterisation of these 'general principles' leads linea recta to a set-theoretical predicate whose extension simply is a theory, so that the models constructed by scientists employing general principles, and therefore obeying these principles, simply are members of that extension. Giere effectively uses theories in full accordance to the structural view, but he no longer wishes to mention what he uses.

${ }^{34}$ Frigg [2006, §2] lists three problems, of which one consists of two problems (our F1, F2, F3, F4 Frigg lumps F3 and F4 together), and two requirements (our F5 and F6).

${ }^{35}$ Sneed's [1979: 135] well-known concept of an empirical claim needs this ontological presupposition too when he wants to claim that a particular concrete actual being, say the solar system, is 'a classical particle-mechanical structure'. Sneed takes the presupposition for granted. 


\section{Opera Consulta}

W. Balzer, C.U. Moulines and J.D. Sneed, An Architectonic for Science: The Structuralist Program, Dordrecht: Reidel, 1987.

R.J. Bogdan (ed.), Patrick Suppes, Dordrecht: Reidel, 1979.

R. Carnap, Philosophical Foundations of Physics. An Introduction to the Philosophy of Science, New York: Basic Books, 1966.

N.C.A. da Costa \& S. French, 'The Model-Theoretic Approach in the Philosophy of Science', Philosophy of Science 57 (1990) 248-265.

N.C.A. da Costa \& R. Chuaqui, 'On Suppes' Set-Theoretical Predicates', Erkenntnis 29 (1988) 95-112.

J.A. Díez, 'A Hundred Years of Numbers. An Historical Introduction to Measurement Theory 1887-1990', Studies in the History and Philosophy of Modern Physics 28 (1997) Part I:167-185, Part II:237-265.

B.C. van Fraassen, The Scientific Image, Oxford: Clarendon Press, 1980.

B.C. van Fraassen, Laws and Symmetries, Oxford: Clarendon Press, 1989.

S. French, 'A Model-Theoretic Account of Representation', Philosophy of Science (PSA Proceedings) 70 (2003) 1472-1483.

S. French \& J. Ladyman, 'Reinflating the Semantic Approach', International Studies in the Philosophy of Science 13.2 (1999) 103-121.

R. Frigg, 'Scientific Representation and the Semantic View of Theories', Theoria 55 (2006) 49-65.

R.N. Giere, Explaining Science. A Cognitive Approach, Chicago \& London: University of Chicago Press, 1988.

R.N. Giere, 'How Models Are Used to Represent Reality', Philosophy of Science 71 (2004) $742-$ 752.

P. Humphreys (ed.), Patrick Suppes: Scientific Philosopher, Volumes 2, Dordrecht: Kluwer, 1994.

W.R. de Jong \& A. Betti, ‘The Classical Model of Science: A Millennia-Old Model of Scientific Rationality', Synthese (2008), this issue.

D.H. Krantz, R.D. Luce, P. Suppes, A. Tversky, Foundations of Measurement, Volume 1, New York: Academic Press, 1971.

M.S. Morgan \& M. Morrison (eds.), Models as Mediators: Perspectives on Natural and Social Science, Cambridge, UK: Cambridge University Press, 1999. 
J.C.C. McKinsey, A.C. Sugar, P. Suppes, 'Axiomatic Foundation of Classical Particle Mechanics', Journal of Rational Mechanics and Analysis 2 (1953) 253-272.

J.C.C. McKinsey \& P. Suppes, 'Transformations of Systems of Classical Particle Mechanics', Journal of Rational Mechanics and Analysis 2 (1953) 272-289.

J.C.C. McKinsey \& P. Suppes, 'On the Notion of Invariance in Classical Mechanics', British Journal for the Philosophy of Science 5 (1955) 290-302.

F.A. Muller, Structures for Everyone, Amsterdam: A. Gerits \& Son, 1998.

F.A. Muller, 'The Deep Black Sea: Observability and Modality Afloat', British Journal for the Philosophy of Science 56 (2005) 61-99.

F.A. Muller \& B.C. van Fraassen, 'How to talk about unobservables', Analysis 68.3 (2008) 1972005.

C.C. O'Brien (ed.), Burke. Relfections on the Revolution in France, Middlesex, England: Penguin Books, 1967.

H. Putnam, 'What theories are not', in E. Nagel, P. Suppes, A. Tarski (eds.), Logic, Methodology and Philosophy of Science: Proceedings of the 1960 International Congress, Stanford, CA: Stanford University Press, 1962, pp. 240-251.

V. Rantala, 'The Old and the New Logic of Metascience', Synthese 39 (1978) 233-247.

J.D. Sneed, The Logical Structure of Mathematical Physics, Dordrecht: Reidel, 1979.

W. Stegmüller, The Structure and Dynamics of Theories, Berlin: Springer-Verlag, 1976.

M. Suárez, 'Scientific representation: against similarity and isomorphism', International Studies in the Philosophy of Science 17.3 (2003) 225-244.

F. Suppe, 'The Search for Philosophic Understanding of Scientific Theories', in: F. Suppe (ed.), The Structure of Scientific Theories, Urbana: University of Illinois Press, 1974, pp. 6-232.

P. Suppes, 'A Comparison of the Meaning and Use of Models in Mathematics and the Empirical Sciences', Synthese 12 (1960) 287-301.

P. Suppes, 'Some Remarks on the Problems and Methods of the Philosophy of Science', Philosophy of Science 21 (1954) 242-248.

P. Suppes, Studies in the Methodology and Foundations of Science, Dordrecht: Reidel, 1961.

P. Suppes, 'The Desirability of Formalisation in Science', Journal of Philosophy 65 (1968) 651-664.

P. Suppes, 'The Structure of Theories and the Analysis of Data', in F. Suppe (ed.), The Structure of Scientific Theories, Urbana: University of Illinois Press, 1974, pp. 266-307. 
P. Suppes, 'Axiomatic Methods in Science', in: Nature, Cognition and System II, M.E. Carvallo (ed.), Dordrecht: Kluwer, 1992, pp. 205-232.

P. Suppes, Models and Methods in the Philosophy of Science, Dordrecht: Kluwer, 1993.

P. Suppes, Representation and Invariance of Scientific Structures, Stanford: Centre for Logic, Language and Computation (distributed by Chicago University Press), 2002.

S. Toulmin, 'The Structure of Scientific Theories', in: F. Suppe (ed.), The Structure of Scientific Theories, Urbana: University of Illinois Press, 1974, pp.600-614. 